Fields Institute Communications

Volume 00, 0000

\title{
Galois corings from the descent theory point of view
}

\author{
S. Caenepeel \\ Faculty of Applied Sciences \\ Vrije Universiteit Brussel, VUB \\ Pleinlaan 2 \\ B-1050 Brussels, Belgium
}

\begin{abstract}
We introduce Galois corings, and give a survey of properties that have been obtained so far. The Definition is motivated using descent theory, and we show that classical Galois theory, Hopf-Galois theory and coalgebra Galois theory can be obtained as a special case.
\end{abstract}

\section{Introduction}

Galois descent theory [36 has many applications in several branches of mathematics, such as number theory, commutative algebra and algebraic geometry; to name such one example, it is an essential tool in computing the Brauer group of a field. In the literature, several generalizations have appeared. Galois theory of commutative rings has been studied by Auslander and Goldman [3] and by Chase, Harrison and Rosenberg [16, see also 21. The group action can be replaced by a Hopf algebra (co)action, leading to Hopf-Galois theory, see [17] (in the case where the Hopf algebra is finitely generated projective), and $\mathbf{2 4}, \mathbf{3 5}$ in the general case. More recently, coalgebra Galois extensions were introduced by Brzeziński and Hajac [10. It became clear recently that a nice unification of all these theories can be formulated using the language of corings. Let us briefly sketch the history.

During the nineties, several unifications of the various kinds of Hopf modules that had appeared in the literature have been proposed. Doi $[22$ and Koppinen 30 introduced Doi-Hopf modules. A more general concept, entwined modules, was proposed by Brzeziński and Majid [11]. Böhm introduced Doi-Hopf modules over a weak bialgebra ([6]), and the author and De Groot proposed weak entwined modules 12. Takeuchi 39] observed that all types of modules can be viewed as comodules over a coring, a concept that was already introduced by Sweedler [38, but then more or less forgotten, at least by Hopf algebra theorists; the idea was further investigated by Brzeziński 9. He generalized several properties that had been studied in special cases to the situation where one works over a general coring, such as separability and Frobenius type properties, and it turned out that computations sometimes become amazingly simple if one uses the language of corings, indicating

1991 Mathematics Subject Classification. Primary 16W30.

Key words and phrases. Coring, Galois extension, descent theory. 
that this is really the right way to look at the problem. Brzeziński also introduces the notion of Galois coring: to a ring extension $i: B \rightarrow A$, one can associate the so-called canonical coring; a morphism from the canonical coring to another coring $\mathcal{C}$ is determined completely by a grouplike element $x$; if this morphism is an isomorphism, then we say that $(\mathcal{C}, x)$ is a Galois coring.

The canonical coring leads to an elegant formulation of descent theory: the category of descent data associated to the extension $i: B \rightarrow A$ is nothing else then the category of comodules over the canonical coring. This is no surprise: to an $A$-coring, we can associate a comonad on the category of $A$-modules, and the canonical coring is exactly the comonad associated to the adjoint pair of functors, given by induction and restriction of scalars. Thus, if a coring is isomorphic to the canonical coring, and if the induction functor is comonadic, then the category of descent data is isomorphic to the category of comodules over this coring, and equivalent to the category of $B$-modules. This unifies all the versions of descent theory that we mentioned at the beginning of this introduction.

In this paper, we present a survey of properties of Galois corings that have been obtained so far. We have organized it as follows: in Section 1 we recall definition, basic properties and examples of comodules over corings; in Section 2 we explain how descent theory can be formulated using the canonical coring. We included a full proof of Proposition 2.3. which is the noncommutative version of the fact that the induction functor is comonadic if and only if the ring morphism is pure as a map of modules. In Section 3 we introduce Galois corings, and discuss some properties, taken from 9 and 41. In Section 4 Morita theory is applied to find some equivalent properties for a progenerator coring to be Galois; in fact, Theorem 4.7 is a new result, and generalizes results of Chase and Sweedler [17. In Section 5 we look at special cases, and we show how to recover the "old" Galois theories. In Section [6] we present a recent generalization, due to El Kaoutit and Gómez Torrecillas [25].

\section{Corings}

Let $A$ be a ring (with unit). The category of $A$-bimodules is a braided monoidal category, and an $A$-coring is by definition a coalgebra in the category of $A$-bimodules. Thus an $A$-coring is a triple $\mathcal{C}=\left(\mathcal{C}, \Delta_{\mathcal{C}}, \varepsilon_{\mathcal{C}}\right)$, where

- $\mathcal{C}$ is an $A$-bimodule;

- $\Delta_{\mathcal{C}}: \mathcal{C} \rightarrow \mathcal{C} \otimes_{A} \mathcal{C}$ is an $A$-bimodule map;

- $\varepsilon_{\mathcal{C}}: \mathcal{C} \rightarrow A$ is an $A$-bimodule map

such that

$$
\left(\Delta_{\mathcal{C}} \otimes_{A} I_{\mathcal{C}}\right) \circ \Delta_{\mathcal{C}}=\left(I_{\mathcal{C}} \otimes_{A} \Delta_{\mathcal{C}}\right) \circ \Delta_{\mathcal{C}},
$$

and

$$
\left(I_{\mathcal{C}} \otimes_{A} \varepsilon_{\mathcal{C}}\right) \circ \Delta_{\mathcal{C}}=\left(\varepsilon_{\mathcal{C}} \otimes_{A} I_{\mathcal{C}}\right) \circ \Delta_{\mathcal{C}}=I_{\mathcal{C}} .
$$

Sometimes corings are considered as coalgebras over noncommutative rings. This point of view is not entirely correct: a coalgebra over a commutative ring $k$ is a $k$-coring, but not conversely: it could be that the left and and right action of $k$ on the coring are different.

The Sweedler-Heyneman notation is also used for a coring $\mathcal{C}$, namely

$$
\Delta_{\mathcal{C}}(c)=c_{(1)} \otimes_{A} c_{(2)},
$$

where the summation is implicitely understood. (1.2) can then be written as

$$
\varepsilon_{\mathcal{C}}\left(c_{(1)}\right) c_{(2)}=c_{(1)} \varepsilon_{\mathcal{C}}\left(c_{(2)}\right)=c .
$$


This formula looks like the corresponding formula for usual coalgebras. Notice however that the order matters in the above formula, since $\varepsilon_{\mathcal{C}}$ now takes values in $A$ which is noncommutative in general. Even worse, the expression $c_{(2)} \varepsilon_{\mathcal{C}}\left(c_{(1)}\right)$ makes no sense at all, since we have no well-defined switch map $\mathcal{C} \otimes_{A} \mathcal{C} \rightarrow \mathcal{C} \otimes_{A} \mathcal{C}$. A morphism between two corings $\mathcal{C}$ and $\mathcal{D}$ is an $A$-bimodule map $f: \mathcal{C} \rightarrow \mathcal{D}$ such that

$$
\Delta_{\mathcal{D}}(f(c))=f\left(c_{(1)}\right) \otimes_{A} f\left(c_{(2)}\right) \text { and } \varepsilon_{\mathcal{D}}(f(c))=\varepsilon_{\mathcal{C}}(c),
$$

for all $c \in \mathcal{C}$. A right $\mathcal{C}$-comodule $M=(M, \rho)$ consists of a right $A$-module $M$ together with a right $A$-linear map $\rho: M \rightarrow M \otimes_{A} \mathcal{C}$ such that:

$$
\left(\rho \otimes_{A} I_{\mathcal{C}}\right) \circ \rho=\left(I_{M} \otimes_{A} \Delta_{\mathcal{C}}\right) \circ \rho,
$$

and

$$
\left(I_{M} \otimes_{A} \varepsilon_{\mathcal{C}}\right) \circ \rho=I_{M} .
$$

We then say that $\mathcal{C}$ coacts from the right on $M$. Left $\mathcal{C}$-comodules and $\mathcal{C}$-bicomodules can be defined in a similar way. We use the Sweedler-Heyneman notation also for comodules:

$$
\rho(m)=m_{[0]} \otimes_{A} m_{[1]} .
$$

(1.4) then takes the form $m_{[0]} \varepsilon_{\mathcal{C}}\left(m_{[1]}\right)=m$. A right $A$-linear map $f: M \rightarrow N$ between two right $\mathcal{C}$-comodules $M$ and $N$ is called right $\mathcal{C}$-colinear if $\rho(f(m))=$ $f\left(m_{[0]}\right) \otimes m_{[1]}$, for all $m \in M$.

Corings were already considered by Sweedler in 38. The interest in corings was revived after a mathematical review written by Takeuchi 39, in which he observed that entwined modules can be considered as comodules over a coring. This will be discussed in the examples below.

Example 1.1 As we already mentioned, if $A$ is a commutative ring, then an $A$-coalgebra is also an $A$-coring.

Example 1.2 Let $i: B \rightarrow A$ be a ring morphism; then $\mathcal{D}=A \otimes_{B} A$ is an $A$-coring. We define

$$
\Delta_{\mathcal{D}}: \mathcal{D} \rightarrow \mathcal{D} \otimes_{A} \mathcal{D} \cong A \otimes_{B} A \otimes_{B} A
$$

and

by

$$
\varepsilon_{\mathcal{D}}: \mathcal{D}=A \otimes_{B} A \rightarrow A
$$

$$
\Delta_{\mathcal{D}}\left(a \otimes_{B} b\right)=\left(a \otimes_{B} 1_{A}\right) \otimes_{A}\left(1_{A} \otimes_{B} b\right) \cong a \otimes_{B} 1_{A} \otimes_{B} b
$$

and

$$
\varepsilon_{\mathcal{D}}\left(a \otimes_{B} b\right)=a b .
$$

Then $\mathcal{D}=\left(\mathcal{D}, \Delta_{\mathcal{D}}, \varepsilon_{\mathcal{D}}\right)$ is an $A$-coring. It is called the canonical coring associated to the ring morphism $i$. We will see in the next section that this coring is crucial in descent theory.

Example 1.3 Let $k$ be a commutative ring, $G$ a finite group, and $A$ a $G$ module algebra. Let $\mathcal{C}=\oplus_{\sigma \in G} A v_{\sigma}$ be the left free $A$-module with basis indexed by $G$, and let $p_{\sigma}: \mathcal{C} \rightarrow A$ be the projection onto the free component $A v_{\sigma}$. We make $\mathcal{C}$ into a right $A$-module by putting

$$
v_{\sigma} a=\sigma(a) v_{\sigma} .
$$

A comultiplication and counit on $\mathcal{C}$ are defined by putting

$$
\Delta_{\mathcal{C}}\left(a v_{\sigma}\right)=\sum_{\tau \in G} a v_{\tau} \otimes_{A} v_{\tau^{-1} \sigma} \text { and } \varepsilon_{\mathcal{C}}=p_{e},
$$


where $e$ is the unit element of $G$. It is straightforward to verify that $\mathcal{C}$ is an $A$ coring. Notice that, in the case where $A$ is commutative, we have an example of an $A$-coring, which is not an $A$-coalgebra, since the left and right $A$-action on $\mathcal{C}$ do not coincide.

Let us give a description of the $\operatorname{right} \mathcal{C}$-comodules. Assume that $M=(M, \rho)$ is a right $\mathcal{C}$-comodule. For every $m \in M$ and $\sigma \in G$, let $\left.\bar{\sigma}(m)=m_{\sigma}=I_{M} \otimes_{A} p_{\sigma}\right)(\rho(m))$. Then we have

$$
\rho(m)=\sum_{\sigma \in G} m_{\sigma} \otimes_{A} v_{\sigma} .
$$

$\bar{e}$ is the identity, since $m=\left(I_{M} \otimes_{A} \varepsilon_{\mathcal{C}}\right) \circ \rho(m)=m_{e}$. Using the coassociativity of the comultiplication, we find

$$
\sum_{\sigma \in G} \rho\left(m_{\sigma}\right) \otimes v_{\sigma}=\sum_{\sigma, \tau \in G} m_{\sigma} \otimes_{A} v_{\tau} \otimes_{A} v_{\tau^{-1} \sigma}=\sum_{\rho, \tau \in G} m_{\tau \rho} \otimes_{A} v_{\tau} \otimes_{A} v_{\rho},
$$

hence $\rho\left(m_{\sigma}\right)=\sum_{\tau \in G} m_{\tau \sigma} \otimes_{A} v_{\tau}$, and $\bar{\tau}(\bar{\sigma}(m))=m_{\tau \sigma}=\overline{\tau \sigma}(m)$, so $G$ acts as a group of $k$-automorphisms on $M$. Moreover, since $\rho$ is right $A$-linear, we have that

$$
\rho(m a)=\sum_{\sigma \in G} \bar{\sigma}(m a) \otimes_{A} v_{\sigma}=\sum_{\sigma \in G} \bar{\sigma}(m) \otimes_{A} v_{\sigma} a=\sum_{\sigma \in G} \bar{\sigma}(m) \sigma(a) \otimes_{A} v_{\sigma}
$$

so $\bar{\sigma}$ is $A$-semilinear (cf. [29 p. 55]): $\bar{\sigma}(m a)=\bar{\sigma}(m) \sigma(a)$, for all $m \in M$ and $a \in A$. Conversely, if $G$ acts as a group of right $A$-semilinear automorphims on $M$, then the formula

$$
\rho(m)=\sum_{\sigma \in G} \bar{\sigma}(m) \otimes_{A} v_{\sigma}
$$

defines a right $\mathcal{C}$-comodule structure on $\mathcal{M}$.

Example 1.4 Now let $k$ be a commutative ring, $G$ an arbitrary group, and $A$ a $G$-graded $k$-algebra. Again let $\mathcal{C}$ be the free left $A$-module with basis indexed by $G$ :

$$
\mathcal{C}=\oplus_{\sigma \in G} A u_{\sigma}
$$

Right $A$-action, comultiplication and counit are now defined by

$$
u_{\sigma} a=\sum_{\tau \in G} a_{\tau} u_{\sigma \tau} ; \Delta_{\mathcal{C}}\left(u_{\sigma}\right)=u_{\sigma} \otimes_{A} u_{\sigma} ; \varepsilon_{\mathcal{C}}\left(u_{\sigma}\right)=1 .
$$

$\mathcal{C}$ is an $A$-coring; let $M=(M, \rho)$ be a right $A$-comodule, and let $M_{\sigma}=\{m \in$ $\left.M \mid \rho(m)=m \otimes_{A} u_{\sigma}\right\}$. It is then clear that $M_{\sigma} \cap M_{\tau}=\{0\}$ if $\sigma \neq \tau$. For any $m \in M$, we can write in a unique way:

$$
\rho(m)=\sum_{\sigma \in G} m_{\sigma} \otimes_{A} u_{\sigma} .
$$

Using the coassociativity, we find that $m_{\sigma} \in M_{\sigma}$, and using the counit property, we find that $m=\sum_{\sigma} m_{\sigma}$. So $M=\oplus_{\sigma \in G} M_{\sigma}$. Finally, if $m \in M_{\sigma}$ and $a \in A_{\tau}$, then it follows from the right $A$-linearity of $\rho$ that

$$
\rho(m a)=\left(m \otimes_{A} u_{\sigma}\right) a=m a \otimes_{A} u_{\sigma \tau},
$$

so $m a \in M_{\sigma \tau}$, and $M_{\sigma} A_{\tau} \subset M_{\sigma \tau}$, and $M$ is a right $G$-graded $A$-module. Conversely, every right $G$-graded $A$-module can be made into a right $\mathcal{C}$-comodule.

Example 1.5 Let $H$ be a bialgebra over a commutative ring $k$, and $A$ a right $H$-comodule algebra. Now take $\mathcal{C}=A \otimes H$, with $A$-bimodule structure

$$
a^{\prime}(b \otimes h) a=a^{\prime} b a_{[0]} \otimes h a_{[1]} .
$$


Now identify $(A \otimes H) \otimes_{A}(A \otimes H) \cong A \otimes H \otimes H$, and define the comultiplication and counit on $\mathcal{C}$, by putting $\Delta_{\mathcal{C}}=I_{A} \otimes \Delta_{H}$ and $\varepsilon_{\mathcal{C}}=I_{A} \otimes \varepsilon_{H}$. Then $\mathcal{C}$ is an $A$-coring. The category $\mathcal{M}^{\mathcal{C}}$ is isomorphic to the category of relative Hopf modules. These are $k$-modules $M$ with a right $A$-action and a right $H$-coaction $\rho$, such that

$$
\rho(m a)=m_{[0]} a_{[0]} \otimes_{A} m_{[1]} a_{[1]}
$$

for all $m \in M$ and $a \in A$.

Example 1.6 Let $k$ be a commutative ring, $A$ a $k$-algebra, and $C$ a $k$-coalgebra, and consider a $k$-linear map $\psi: C \otimes A \rightarrow A \otimes C$. We use the following Sweedler type notation, where the summation is implicitely understood:

$$
\psi(c \otimes a)=a_{\psi} \otimes c^{\psi}=a_{\Psi} \otimes c^{\Psi} .
$$

$(A, C, \psi)$ is called a (right-right) entwining structure if the four following conditions are satisfied:

$$
\begin{aligned}
& (a b)_{\psi} \otimes c^{\psi}=a_{\psi} b_{\Psi} \otimes c^{\psi \Psi} \\
& \left(1_{A}\right)_{\psi} \otimes c^{\psi}=1_{A} \otimes c \\
& a_{\psi} \otimes \Delta_{C}\left(c^{\psi}\right)=a_{\psi \Psi} \otimes c_{(1)}^{\Psi} \otimes c_{(2)}^{\psi} \\
& \varepsilon_{C}\left(c^{\psi}\right) a_{\psi}=\varepsilon_{C}(c) a .
\end{aligned}
$$

Let $\mathcal{C}=A \otimes C$ as a $k$-module, with $A$-bimodule structure

$$
a^{\prime}(b \otimes c) a=a^{\prime} b a_{\psi} \otimes c^{\psi} .
$$

Comultiplication and counit on $A \otimes C$ are defined as in Example $1.5 \mathcal{C}$ is a coring, and the category $\mathcal{M}^{\mathcal{C}}$ is isomorphic to the category $\mathcal{M}(\psi)_{A}^{C}$ of entwined modules. These are $k$-modules $M$ with a right $A$-action and a right $C$-coaction $\rho$ such that

$$
\rho(m a)=m_{[0]} a_{\psi} \otimes_{A} m_{[1]}^{\psi},
$$

for all $m \in M$ and $a \in A$.

Actually Examples 1.3 1.4 and 1.5 are special cases of Example 1.6

- Example 1.3 take $C=(k G)^{*}=\oplus_{g \in G} k v_{\sigma}$, the dual of the group $\operatorname{ring} k G$, and $\psi\left(v_{\sigma} \otimes a\right)=\sigma(a) \otimes v_{\sigma}$

- Example 1.4 take $C=k G=\oplus_{g \in G} k u_{\sigma}$, the group ring, and $\psi\left(u_{\sigma} \otimes a\right)=$ $\sum_{\tau \in G} a_{\tau} \otimes u_{\sigma \tau}$

- Example 1.5 take $C=H$, and $\psi(h \otimes a)=a_{[0]} \otimes h a_{[1]}$.

If $\mathcal{C}$ is an $A$-coring, then its left dual ${ }^{*} \mathcal{C}={ }_{A} \operatorname{Hom}(\mathcal{C}, A)$ is a ring, with (associative) multiplication given by the formula

$$
f \# g=g \circ\left(I_{\mathcal{C}} \otimes_{A} f\right) \circ \Delta_{\mathcal{C}} \text { or }(f \# g)(c)=g\left(c_{(1)} f\left(c_{(2)}\right)\right),
$$

for all left $A$-linear $f, g: \mathcal{C} \rightarrow A$ and $c \in \mathcal{C}$. The unit is $\varepsilon_{\mathcal{C}}$. We have a ring homomorphism $i: A \rightarrow{ }^{*} \mathcal{C}, i(a)(c)=\varepsilon_{\mathcal{C}}(c) a$. We easily compute that

$$
(i(a) \# f)(c)=f(c a) \text { and }(f \# i(a))(c)=f(c) a,
$$

for all $f \in{ }^{*} \mathcal{C}, a \in A$ and $c \in \mathcal{C}$. We have a functor $F: \mathcal{M}^{\mathcal{C}} \rightarrow \mathcal{M}^{*} \mathcal{C}$, where $F(M)=M$ as a right $A$-module, with right ${ }^{*} \mathcal{C}$-action given by $m \cdot f=m_{[0]} f\left(m_{[1]}\right)$, for all $m \in M, f \in{ }^{*} \mathcal{C}$. If $\mathcal{C}$ is finitely generated and projective as a left $A$-module, then $F$ is an isomorphism of categories: given a right ${ }^{*} \mathcal{C}$-action on $M$, we recover the right $\mathcal{C}$-coaction by putting $\rho(m)=\sum_{j}\left(m \cdot f_{j}\right) \otimes_{A} c_{j}$, where $\left\{\left(c_{j}, f_{j}\right) \mid j=1, \cdots, n\right\}$ is a finite dual basis of $\mathcal{C}$ as a left $A$-module. ${ }^{*} \mathcal{C}$ is a right $A$-module, by (1.10): $(f \cdot a)(c)=f(c) a$, and we can consider the double dual $\left({ }^{*} \mathcal{C}\right)^{*}=\operatorname{Hom}_{A}\left({ }^{*} \mathcal{C}, A\right)$. We 
have a canonical morphism $i: \mathcal{C} \rightarrow\left({ }^{*} \mathcal{C}\right)^{*}, i(c)(f)=f(c)$, and we call $\mathcal{C}$ reflexive (as a left $A$-module) if $i$ is an isomorphism. If $\mathcal{C}$ is finitely generated projective as a left $A$-module, then $\mathcal{C}$ is reflexive. For any $\varphi \in\left({ }^{*} \mathcal{C}\right)^{*}$, we then have that $\varphi=i\left(\sum_{j} \varphi\left(f_{j}\right) c_{j}\right)$.

Corings with a grouplike element. Let $\mathcal{C}$ be an $A$-coring, and suppose that $\mathcal{C}$ coacts on $A$. Then we have a map $\rho: A \rightarrow A \otimes_{A} \mathcal{C} \cong \mathcal{C}$. The fact that $\rho$ is right $A$-linear implies that $\rho$ is completely determined by $\rho\left(1_{A}\right)=x: \rho(a)=x a$. The coassociativity of the coaction yields that $\Delta_{\mathcal{C}}(x)=x \otimes_{A} x$ and the counit property gives us that $\varepsilon_{\mathcal{C}}(x)=1_{A}$. We say that $x$ is a grouplike element of $\mathcal{C}$ and we denote $G(\mathcal{C})$ for the set of all grouplike elements of $\mathcal{C}$. If $x \in G(\mathcal{C})$ is grouplike, then the associated $\mathcal{C}$-coaction on $A$ is given by $\rho(a)=x a$.

If $x \in G(\mathcal{C})$, then we call $(\mathcal{C}, x)$ a coring with a fixed grouplike element. For $M \in \mathcal{M}^{\mathcal{C}}$, we call

$$
M^{\mathrm{coC}}=\left\{m \in M \mid \rho(m)=m \otimes_{A} x\right\}
$$

the submodule of coinvariants of $M$; note that this definition depends on the choice of the grouplike element. Also observe that

$$
A^{\mathrm{coC}}=\{b \in A \mid b x=x b\}
$$

is a subring of $A$. Let $i: B \rightarrow A$ be a ring morphism. $i$ factorizes through $A^{\text {coC }}$ if and only if

$$
x \in G(\mathcal{C})^{B}=\{x \in G(\mathcal{C}) \mid x b=b x, \text { for all } b \in B\} .
$$

We then have two pairs of adjoint functors $(F, G)$ and $\left(F^{\prime}, G^{\prime}\right)$, respectively between the categories $\mathcal{M}_{B}$ and $\mathcal{M}^{\mathcal{C}}$ and the categories ${ }_{B} \mathcal{M}$ and ${ }^{\mathcal{C}} \mathcal{M}$. For $N \in \mathcal{M}_{B}$ and $M \in \mathcal{M}^{\mathcal{C}}$,

$$
F(N)=N \otimes_{B} A \text { and } G(M)=M^{\mathrm{coC}} .
$$

The unit and counit of the adjunction are

$$
\begin{gathered}
\nu_{N}: N \rightarrow\left(N \otimes_{B} A\right)^{\mathrm{coC}}, \nu_{N}(n)=n \otimes_{B} 1 ; \\
\zeta_{M}: M^{\mathrm{coC}} \otimes_{B} A \rightarrow M, \zeta_{M}\left(m \otimes_{B} a\right)=m a .
\end{gathered}
$$

The other adjunction is defined in a similar way. We want to discuss when $(F, G)$ and $\left(F^{\prime}, G^{\prime}\right)$ are category equivalences. In Section 2 we will do this for the canonical coring associated to a ring morphism; we will study the general case in Section 3

\section{The canonical coring and descent theory}

Let $i: B \rightarrow A$ be a ring morphism. The problem of descent theory is the following: suppose that we have a right $A$-module $M$. When do we have a right $B$-module $N$ such that $N=M \otimes_{B} A$ ? The same problem can be stated for modules with an additional structure, for example algebras. In the case where $A$ and $B$ are commutative, this problem has been discussed in a purely algebraic context in [29. In fact the results in 29 are the affine versions of Grothendieck's descent theory for schemes, see $\mathbf{2 8}$. In the situation where $A$ and $B$ are arbitrary, descent theory has been discussed by Cipolla [18, and, more recently, by Nuss $\mathbf{3 3}$. For a purely categorical treatment of the problem, making use of monads, we refer to [7. Here we will show that the results in 29] and 18 can be restated elegantly in terms of comodules over the canonical coring.

Let $\mathcal{D}=A \otimes_{B} A$ be the canonical coring associated to the ring morphism $i: B \rightarrow A$, and let $M=(M, \rho)$ be a right $\mathcal{D}$-comodule. We will identify $M \otimes_{A} \mathcal{D} \cong$ 
$M \otimes_{B} A$ using the natural isomorphism. The coassociativity and the counit property then take the form

$$
\rho\left(m_{[0]}\right) \otimes m_{[1]}=m_{[0]} \otimes_{B} 1_{A} \otimes_{B} m_{[1]} \text { and } m_{[0]} m_{[1]}=m .
$$

$1_{A} \otimes_{B} 1_{A}$ is a grouplike element of $\mathcal{D}$. As we have seen at the end of Section 1 we have two pairs of adjoint functors, respectively between $\mathcal{M}_{B}$ and $\mathcal{M}^{\mathcal{D}}$, and ${ }_{B} \mathcal{M}$ and ${ }^{\mathcal{D}} \mathcal{M}$, which we will denote by $(K, R)$ and $\left(K^{\prime}, R^{\prime}\right)$. The unit and counit of the adjunction will be denoted by $\eta$ and $\varepsilon$. $K$ is called the comparison functor. If $(K, R)$ is an equivalence of categories, then the "descent problem" is solved: $M \in \mathcal{M}_{A}$ is isomorphic to some $N \otimes_{B} A$ if and only if we can define a right $\mathcal{D}$-coaction on $M$.

Recall that a morphism of left $B$-modules $f: M \rightarrow M^{\prime}$ is called pure if and only if $f_{N}=I_{N} \otimes_{B} f: N \otimes_{B} M \rightarrow N \otimes_{B} M^{\prime}$ is monic, for every $N \in \mathcal{M}_{B} . i: B \rightarrow A$ is pure as a morphism of left $B$-modules if and only if $\eta_{N}$ being injective, for all $N \in \mathcal{M}_{B}$, since $\eta_{N}$ factorizes through $i_{N}$.

Proposition 2.1 The comparison functor $K$ is fully faithful if and only if $i: B \rightarrow A$ is pure as a morphism of left B-modules.

Proof The comparison functor $K$ is fully faithful if and only if $\eta_{N}$ is bijective, for all $N \in \mathcal{M}_{B}$. From the above observation, it follows that it suffices to show that left purity of $i$ implies that $\eta_{N}$ is surjective. Since $\eta_{N}$ is injective, we have that $N \subset\left(N \otimes_{B} A\right)^{\mathrm{coD}} \subset N \otimes_{B} A$. Take $q=\sum_{i} n_{i} \otimes_{B} a_{i} \in\left(N \otimes_{B} A\right)^{\mathrm{co} \mathcal{D}}$. Then

$$
\rho\left(\sum_{i} n_{i} \otimes_{B} a_{i}\right)=\sum_{i} n_{i} \otimes_{B} a_{i} \otimes_{B} 1=\sum_{i} n_{i} \otimes_{B} 1 \otimes_{B} a_{i} .
$$

Consider the right $B$-module $P=\left(P \otimes_{B} A\right) / N$, and let $\pi: N \otimes_{B} A \rightarrow P$ be the canonical projection. Applying $\pi \otimes_{B} I_{A}$ to (2.1), we obtain

$$
\pi(q) \otimes_{B} 1=\sum_{i} \pi\left(n_{i} \otimes_{B} 1\right) \otimes_{B} a_{i}=0 \text { in } P \otimes_{B} A,
$$

hence $\pi(q)=0$, since $i_{P}$ is an injection. This means that $q \in N$, which is exactly what we needed.

We also have an easy characterization of the fact that $R$ is fully faithful.

Proposition 2.2 The right adjoint $R$ of the comparison functor $K$ is fully faithful if and only if $\bullet \otimes_{B} A$ preserves the equalizer of $\rho$ and $i_{M}$, for every $(M, \rho) \in$ $\mathcal{M}^{\mathcal{D}}$. In particular, if $A$ is flat as a left $B$-module, then $R$ is fully faithful.

Proof $M^{\mathrm{co} \mathcal{D}}$ is the equalizer of the maps

$$
0 \longrightarrow M^{\mathrm{coD}} \stackrel{j}{\longrightarrow} M \underset{i_{M}}{\stackrel{\rho}{\longrightarrow}} M \otimes_{B} A
$$

First assume that $M^{\mathrm{coD}} \otimes_{B} A$ is the equalizer

$$
0 \longrightarrow M^{\operatorname{coD}} \otimes_{B} A \stackrel{j \otimes_{B} I_{A}}{\longrightarrow} M \otimes_{B} A \underset{i_{M} \otimes_{B} I_{A}}{\stackrel{\rho \otimes_{B} I_{A}}{\longrightarrow}} M \otimes_{B} A \otimes_{B} A
$$

From the coassociativity of $\rho$, it now follows that $\rho(m) \in M^{\mathrm{coD}} \otimes_{B} A \subset M \otimes_{B} A \cong$ $M \otimes_{A}\left(A \otimes_{B} A\right)$, for all $m \in M$, and we have a map $\rho: M \rightarrow M^{\mathrm{coD}} \otimes_{B} A$. From the counit property, it follows that $\varepsilon_{M} \circ \rho=I_{M}$. For $m \in M^{\mathrm{coD}}$ and $a \in A$, we have

$$
\rho\left(\varepsilon_{M}\left(m \otimes_{B} a\right)\right)=\rho(m a)=\rho(m) a=\left(m \otimes_{B} 1\right) a=m \otimes_{B} a .
$$

Thus the counit $\varepsilon_{M}$ has an inverse, for all $M$, and $R$ is fully faithful. 
Conversely, assume that $\varepsilon_{M}$ is bijective. Take $\sum_{i} m_{i} \otimes_{B} a_{i} \in M^{\mathrm{co} \mathcal{D}} \otimes_{B} A$, and put $m=\sum_{i} m_{i} a_{i} \in M$. Then $\rho(m)=m_{[0]} \otimes_{B} m_{[1]}=\sum_{i} m_{i} \otimes_{B} a_{i} \in$ $M \otimes_{B} A$. Consequently, if $\sum_{i} m_{i} \otimes_{B} a_{i}=0 \in M \otimes_{B} A$, then $m=m_{[0]} m_{[1]}=0$, so $\sum_{i} m_{i} \otimes_{B} a_{i}=0 \in M^{\mathrm{coD}} \otimes_{B} A$, and we have shown that the canonical map $M^{\mathrm{coD}} \otimes_{B} A \rightarrow M \otimes_{B} A$ is injective.

If $A$ and $B$ are commutative, and $i: B \rightarrow A$ is pure as a morphism of $B$ modules, then $\bullet \otimes_{B} A$ preserves the equalizer of $\rho$ and $i_{M}$, for every $(M, \rho) \in$ $\mathcal{M}^{\mathcal{D}}$, and therefore $R$ is fully faithful. This result is due to Joyal and Tierney (unpublished); an elementary proof was given recently by Mesablishvili [32. We will now adapt Mesablishvili's proof to the noncommutative situation. In view of Proposition 2.1] one would expect that a sufficient condition for the fully faithfulness of $R$ is the fact that $i$ is pure as a morphism of left $B$-modules. It came as a surprise to the author that we need right purity instead of left purity.

We consider the contravariant functor $C=\operatorname{Hom}_{\mathbb{Z}}(\bullet, \mathbb{Q} / \mathbb{Z}): \underline{\underline{\mathrm{Ab}}} \rightarrow \underline{\underline{\mathrm{Ab}}} . \mathbb{Q} / \mathbb{Z}$ is an injective cogenerator of $\underline{\underline{\mathrm{Ab}}}$, and therefore $C$ is exact and reflects isomorphisms. If $B$ is a ring, then $C$ induces functors

$$
C: \mathcal{M}_{B} \rightarrow{ }_{B} \mathcal{M} \text { and }{ }_{B} \mathcal{M} \rightarrow \mathcal{M}_{B} .
$$

For example, if $M \in \mathcal{M}_{B}$, then $C(M)$ is a left $B$-module, by putting $(b \cdot f)(m)=$ $f(m b)$. For $M \in \mathcal{M}_{B}$ and $P \in{ }_{B} M$, we have the following isomorphisms, natural in $M$ and $P$ :

$$
\operatorname{Hom}_{B}(M, C(P)) \cong{ }_{B} \operatorname{Hom}(P, C(M)) \cong C\left(M \otimes_{B} P\right)
$$

If $P \in{ }_{B} \mathcal{M}_{B}$, then $C(P) \in{ }_{B} \mathcal{M}_{B}$, and the above isomorphisms are isomorphisms of left $B$-modules.

Proposition 2.3 Let $i: B \rightarrow A$ be a ring morphism, and assume that $i$ is pure as a morphism of right $B$-modules. Then the adjoint $R$ of the comparison functor is fully faithful.

Proof We have to show that (2.2) is exact, for all $(M, \rho) \in \mathcal{M}^{\mathcal{D}}$. If $i$ is pure in $\mathcal{M}_{B}$, then $i_{C(B)}: B \otimes_{B} C(B) \rightarrow A \otimes_{B} C(B)$ is a monomorphism in $\mathcal{M}_{B}$, hence

$$
C\left(i_{C(B)}\right): C\left(A \otimes_{B} C(B)\right) \rightarrow C\left(B \otimes_{B} C(B)\right)
$$

is an epimorphism in ${ }_{B} \mathcal{M}$. Applying (2.3), we find that

$$
C(i) \circ \bullet:{ }_{B} \operatorname{Hom}(C(B), C(A)) \rightarrow{ }_{B} \operatorname{Hom}(C(B), C(B))
$$

is also an epimorphism. This implies that $C(i): C(A) \rightarrow C(B)$ is a split epimorphism in ${ }_{B} \mathcal{M}$, and then it follows that for every $M \in \mathcal{M}_{B}$,

$$
C(i) \circ \bullet: \operatorname{Hom}_{B}(M, C(A)) \rightarrow \operatorname{Hom}_{B}(M, C(B))
$$

is a split epimorphism in ${ }_{B} \mathcal{M}$. Applying (2.3) again, we find that

$$
C\left(i_{M}\right): C\left(M \otimes_{B} A\right) \rightarrow C(M)
$$


is a split epimorphism in ${ }_{B} \mathcal{M}$.

In $\mathcal{M}_{B}$, we have the following commutative diagram with exact rows:

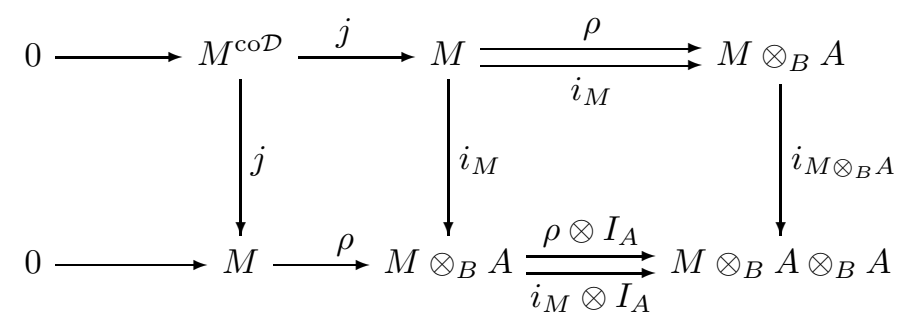

Applying the functor $C$ to this diagram, we obtain the following commutative diagram with exact rows. We also know that $C\left(i_{M}\right)$ and $C\left(i_{M \otimes_{B} A}\right)$ have right inverses $h$ and $h^{\prime}$.

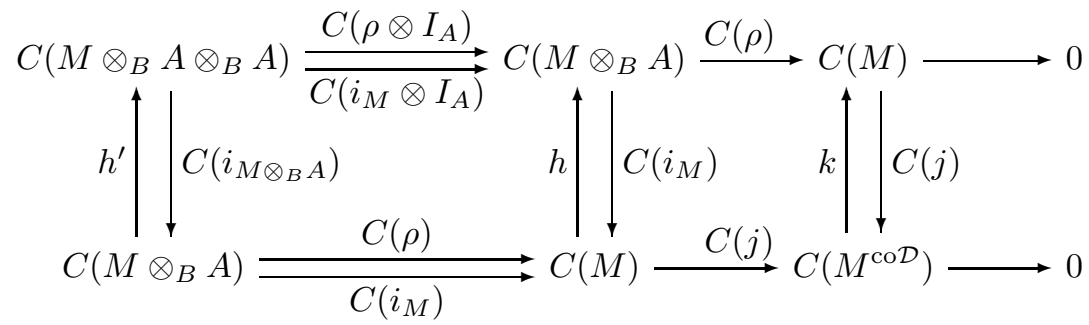

Diagram chasing leads to the existence of a right inverse $k$ of $C(j)$, such that $k \circ C(j)=C(\rho) \circ h$. But this means that the bottom row of the above diagram is a split fork in ${ }_{B} \mathcal{M}$, split by the morphisms

$$
C\left(M \otimes_{B} A\right) \stackrel{h}{\longleftarrow} C(M) \stackrel{k}{\longleftarrow} C\left(M^{\mathrm{coD}}\right)
$$

(see [31 p.149] for the definition of a split fork). Split forks are preserved by arbitrary functors, so applying ${ }_{B} \operatorname{Hom}(A, \bullet)$, we obtain a split fork in ${ }_{B} \mathcal{M}$; using (2.3), we find that this split fork is isomorphic to

$$
C\left(M \otimes_{B} A \otimes_{B} A\right) \underset{\overrightarrow{C\left(i_{M} \otimes I_{A}\right)}}{C} C\left(M \otimes_{B} A\right) \stackrel{C\left(j \otimes I_{A}\right)}{\longrightarrow} C\left(M^{\mathrm{coD}} \otimes_{B} A\right)
$$

The functor $C$ is exact and reflects isomorphisms, hence it also reflects coequalizers. It then follows that (2.2) is an equalizer in $\mathcal{M}_{B}$, as needed.

The converse of Proposition 2.3] is not true in general: the natural inclusion $i: \mathbb{Z} \rightarrow \mathbb{Q}$ is not pure in $\mathcal{M}_{\mathbb{Z}}$, but the functor $R$ is fully faithful. Indeed, if $(M, \rho) \in \mathcal{M}^{\mathcal{D}}$, then $M$ is a $\mathbb{Q}$-vector space, and $\rho: M \rightarrow M \otimes_{\mathbb{Z}} \mathbb{Q} \cong M$ is the identity, $M^{\text {coD }}=M$, and $\varepsilon_{M}: M^{\text {coD }} \otimes_{\mathbb{Z}} \mathbb{Q} \cong M \rightarrow M$ is also the identity.

It would be interesting to know if there exists a ring morphism $i: B \rightarrow A$ which is pure in ${ }_{B} \mathcal{M}$, but not in $\mathcal{M}_{B}$, and such that $(K, R)$ is an equivalence of categories.

Consider $K^{\prime}=A \otimes_{B} \bullet:{ }_{B} \mathcal{M} \rightarrow{ }^{\mathcal{D}} \mathcal{M}$ and $R^{\prime}={ }^{\operatorname{co} \mathcal{D}}(\bullet):{ }^{\mathcal{D}} \mathcal{M} \rightarrow{ }_{B} \mathcal{M}$. The next result is an immediate consequence of Propositions 2.2 and 2.3 and their left handed versions, and can be viewed as the noncommutative version of the Joyal-Tierney Theorem.

Theorem 2.4 Let $i: B \rightarrow A$ be a morphism of rings. Then the following assertions are equivalent.

1. $(K, R)$ and $\left(K^{\prime}, R^{\prime}\right)$ are equivalences of categories;

2. $K$ and $K^{\prime}$ are fully faithful; 
3. $i$ is pure in $\mathcal{M}_{B}$ and ${ }_{B} \mathcal{M}$.

We have seen in Proposition 2.2 that $R$ is fully faithful if $A$ is flat as a left $B$-module.

Proposition 2.5 Let $i: B \rightarrow A$ be a morphism of rings, and assume that $A$ is flat as a left $B$-module. Then $(K, R)$ is an equivalence of categories if and only if $A$ is faithfully flat as a left $B$-module.

Proof First assume that $A$ is faithfully flat as a left $B$-module. It follows from Proposition 2.1] that it suffices to show that $A$ is pure as a left $B$-module. For $N \in \mathcal{M}_{B}$, the map

$$
f=i_{N} \otimes_{B} I_{A}: N \otimes_{B} A \rightarrow N \otimes_{B} A \otimes_{B} A
$$

is injective: if $f\left(\sum_{i} n_{i} \otimes_{B} a_{i}\right)=\sum_{i} n_{i} \otimes_{B} 1 \otimes_{B} a_{i}=0$, then, multiplying the second and third tensor fact, we find that $\sum_{i} n_{i} \otimes_{B} a_{i}=0$. Since $A$ is faithfully flat as a left $B$-module, it follows that $i_{N}$ is injective.

Conversely assume that $(K, R)$ is an equivalence of categories. Then the functor $R$ is exact. Let

$$
0 \rightarrow N^{\prime} \rightarrow N \rightarrow N^{\prime \prime} \rightarrow 0
$$

be a sequence of right $B$-modules such that the sequence

$$
0 \rightarrow N^{\prime} \otimes_{B} A \rightarrow N \otimes_{B} A \rightarrow N^{\prime \prime} \otimes_{B} A \rightarrow 0
$$

is exact. Applying the functor $R$ to the sequence, and using the fact that $\eta$ is an isomorphism, we find that (2.4) is exact, so it follows that $A$ is faithfully flat as a left $B$-module.

The descent data that are considered in [18 are nothing else then comodules over the canonical coring (although the author of 18 was not aware of this). The descent data in [29] are different, so let us indicate how to go from descent data to comodules over the canonical coring.

Let $i: B \rightarrow A$ be a morphism of commutative rings. A descent datum consists of a pair $(M, g)$, with $M \in \mathcal{M}_{A}$, and $g: A \otimes_{B} M \rightarrow M \otimes_{B} A$ an $A \otimes_{B} A$-module homorphism such that

$$
g_{2}=g_{3} \circ g_{1}: A \otimes_{B} A \otimes_{B} M \rightarrow A \otimes_{B} M \otimes_{B} A
$$

and

$$
\mu_{M}\left(g\left(1 \otimes_{B} m\right)\right)=m,
$$

for all $m \in M$. Here $g_{i}$ is obtained by applying $I_{A}$ to the $i$-th tensor position, and $g$ to the two other ones. It can be shown that (2.6) can be replaced by the condition that $g$ is a bijection. A morphism of two descent data $(M, g)$ and $\left(M^{\prime}, g^{\prime}\right)$ consists of an $A$-module homomorphism $f: M \rightarrow M^{\prime}$ such that

$$
\left(f \otimes_{B} I_{A}\right) \circ g=g^{\prime} \circ\left(I_{A} \otimes_{B} f\right) .
$$

$\underline{\underline{\operatorname{Desc}}}(A / B)$ will be the category of descent data.

Proposition 2.6 Let $i: B \rightarrow A$ be a morphism of commutative rings. We have an isomorphism of categories

$$
\underline{\underline{\operatorname{Desc}}}(A / B) \cong \mathcal{M}^{A \otimes_{B} A}
$$


Proof (sketch) For a right $\mathcal{D}$-comodule $(M, \rho)$, we define $g: A \otimes_{B} M \rightarrow$ $M \otimes_{B} A$ by $g\left(a \otimes_{B} m\right)=m_{[0]} a \otimes_{B} m_{[1]}$. Then $(M, g)$ is a descent datum. Conversely, given a descent datum $(M, g)$, the map $\rho: M \rightarrow M \otimes_{B} A, \rho(m)=g\left(1 \otimes_{B} m\right)$ makes $M$ into a right $\mathcal{D}$-comodule.

\section{Galois corings}

Let $A$ be a ring, $(\mathcal{C}, x)$ a coring with a fixed grouplike element, and $i: B \rightarrow A^{\text {coC }}$ a ring morphism. We have seen at the end of Section 1 that we have two pairs of adjoint functors $(F, G)$ and $\left(F^{\prime}, G^{\prime}\right)$. We also have a morphism of corings

$$
\text { can : } \mathcal{D}=A \otimes_{B} A \rightarrow \mathcal{C}, \operatorname{can}\left(a \otimes_{B} a^{\prime}\right)=a x a^{\prime} .
$$

Proposition 3.1 With notation as above, we have the following results.

1. If $F$ is fully faithful, then $i: B \rightarrow A^{\mathrm{coC}}$ is an isomorphism;

2. if $G$ is fully faithful, then can : $\mathcal{D}=A \otimes_{B} A \rightarrow \mathcal{C}$ is an isomorphism.

Proof 1. $F$ is fully faithful if and only if $\nu$ is an isomorphism; it then suffices to observe that $i=\nu_{B}$.

2. $G$ is fully faithful if and only if $\zeta$ is an isomorphism. $\mathcal{C} \in \mathcal{M}^{\mathcal{C}}$, the right coaction being induced by the comultiplication. The map $f: A \rightarrow \mathcal{C}^{\mathrm{coC}}, f(a)=a x$, is an isomorphism of $(A, B)$-bimodules; the inverse of $f$ is the restriction of $\varepsilon_{\mathcal{C}}$ to $\mathcal{C}^{\text {coC }}$. Indeed, if $c \in \mathcal{C}^{\mathrm{co} \mathcal{C}}$, then $\Delta_{\mathcal{C}}(c)=c \otimes_{A} x$, hence $c=\varepsilon(c) x=f(\varepsilon(c))$. It follows that can $=\zeta_{\mathcal{C}} \circ\left(f \otimes_{B} I_{A}\right)$ is an isomorphism.

Proposition 3.1 leads us to the following Definition.

Definition 3.2 Let $(\mathcal{C}, x)$ be an $A$-coring with a fixed grouplike, and let $B=$ $A^{\text {coC }}$. We call $(\mathcal{C}, x)$ a Galois coring if the canonical coring morphism can : $\mathcal{D}=$ $A \otimes_{B} A \rightarrow \mathcal{C}, \operatorname{can}\left(a \otimes_{B} b\right)=a x b$ is an isomorphism.

Let $i: B \rightarrow A$ be a ring morphism. If $x \in G(\mathcal{C})^{B}$, then we can define a functor

$$
\Gamma: \mathcal{M}^{\mathcal{D}} \rightarrow \mathcal{M}^{\mathcal{C}}, \Gamma(M, \rho)=(M, \widetilde{\rho})
$$

with $\widetilde{\rho}(m)=m_{[0]} \otimes_{A} x m_{[1]} \in M \otimes_{A} \mathcal{C}$ if $\rho(m)=m_{[0]} \otimes_{B} m_{[1]} \in M \otimes_{B} A$. It is easy to see that $\Gamma \circ K=F$, and therefore we have the following result.

Proposition 3.3 Let $(\mathcal{C}, x)$ be a Galois A-coring. Then $\Gamma$ is an isomorphism of categories. Consequently $R$ (resp. K) is fully faithful if and only if $G$ (resp. F) is fully faithful.

Let us now give some alternative characterizations of Galois corings; for the proof, we refer to [41, 3.6].

Proposition 3.4 Let $(\mathcal{C}, x)$ be an A-coring with fixed grouplike element, and $B=A^{\mathrm{coC}}$. The following assertions are equivalent.

1. $(\mathcal{C}, x)$ is Galois;

2. if $(M, \rho) \in \mathcal{M}^{\mathcal{C}}$ is such that $\rho: M \rightarrow M \otimes_{A} \mathcal{C}$ is a coretraction, then the evaluation map

$$
\varphi_{M}: \operatorname{Hom}^{\mathcal{C}}(A, M) \otimes_{B} A \rightarrow M, \varphi_{M}\left(f \otimes_{B} m\right)=f(m)
$$

is an isomorphism;

3. $\varphi_{\mathcal{C}}$ is an isomorphism.

From Theorem 2.4 and Proposition 3.3. we immediately obtain the following result. 
Theorem 3.5 Let $(\mathcal{C}, x)$ be a Galois A-coring, and put $B=A^{\mathrm{coC}}$. Then the following assertions are equivalent.

1. $(F, G)$ and $\left(F^{\prime}, G^{\prime}\right)$ are equivalences of categories;

2. the functors $F$ and $F^{\prime}$ are fully faithful;

3. $i: B \rightarrow A$ is pure in ${ }_{B} \mathcal{M}$ and $\mathcal{M}_{B}$.

Remark 3.6 Let us make some remarks about terminology. In the literature, there is an inconsistency in the use of the term "Galois". An alternative definition is to require that $(\mathcal{C}, x)$ satisfies the equivalent definitions of Theorem 3.5] so that $(F, G)$ and $\left(F^{\prime}, G^{\prime}\right)$ are category equivalences. In Section $[5$ we will discuss special cases that have appeared in the literature before. In some cases, there is an agreement with Definition 3.2 (see e.g. [8] [35), while in other cases, category equivalence is required (see e.g. [17, [21]).

In the particular situation where $\mathcal{C}=A \otimes H$, as in Example 1.5 the property that $(F, G)$ is an equivalence (resp. $G$ is fully faithful) is called the Strong (resp. Weak) Structure Theorem (see [24]). Let $i: B \rightarrow A$ be a ring morphism, and $\mathcal{D}$ the canonical coring. In the situation where $A$ and $B$ are commutative, $i$ is called a descent morphism (resp. an effective descent morphism) if $K$ is fully faithful (resp. $(K, R)$ is an equivalence). In the general situation, $(K, R)$ is an equivalence if and only if the functor $\bullet \otimes_{B} A: \mathcal{M}_{B} \rightarrow \mathcal{M}_{A}$ is comonadic (see e.g. 7 . Ch. 4]).

Let us next look at the case where $A$ is flat as a left $B$-module. Wisbauer 41 ] calls the following two results the Galois coring Structure Theorem.

Proposition 3.7 Let $(\mathcal{C}, x)$ be an A-coring with fixed grouplike element, and $B=A^{\mathrm{coC}}$. Then the following statements are equivalent.

1. $(\mathcal{C}, x)$ is Galois and $A$ is flat as a left B-module;

2. $G$ is fully faithful and $A$ is flat as a left B-module;

3. $\mathcal{C}$ is flat as a left $A$-module, and $A$ is a generator in $\mathcal{M}^{\mathcal{C}}$.

Proof 1) $\Rightarrow$ 2) follows from Propositions 2.2 and 3.3 2) $\Rightarrow$ 1) follows from

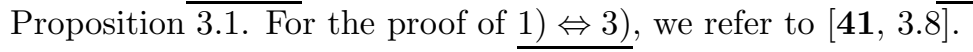

Proposition 3.8 Let $(\mathcal{C}, x)$ be an A-coring with fixed grouplike element, and $B=A^{\mathrm{coC}}$. Then the following statements are equivalent.

1. $(\mathcal{C}, x)$ is Galois and $A$ is faithfully flat as a left B-module;

2. $(F, G)$ is an equivalence and $A$ is flat as a left B-module;

3. $\mathcal{C}$ is flat as a left $A$-module, and $A$ is a projective generator in $\mathcal{M}^{\mathcal{C}}$.

Proof The equivalence of 1) and 2) follows from Propositions 2.5 and 3.3 For the remaining equivalence, we refer to 41 .

A right $\mathcal{C}$-comodule $N$ is called semisimple (resp. simple) in $\mathcal{M}^{\mathcal{C}}$ if every $\mathcal{C}$-monomorphism $U \rightarrow N$ is a coretraction (resp. an isomorphism). Similar definitions apply to left $\mathcal{C}$-comodules and $(\mathcal{C}, \mathcal{C})$-bicomodules. $\mathcal{C}$ is said to be right (left) semisimple if it is semisimple as a right (left) $\mathcal{C}$-comodule. $\mathcal{C}$ is called a simple coring if it is simple as a $(\mathcal{C}, \mathcal{C})$-bicomodule. For the proof of the following result, we refer to $2 \mathbf{2 6}$.

Proposition 3.9 For an $A$-coring $\mathcal{C}$, the following assertions are equivalent:

1. $\mathcal{C}$ is right semisimple;

2. $\mathcal{C}$ is projective as a left $A$-module and $\mathcal{C}$ is semisimple as a left ${ }^{*} \mathcal{C}$-module;

3. $\mathcal{C}$ is projective as a right $A$-module and $\mathcal{C}$ is semisimple as a right $\mathcal{C}^{*}$-module; 
4. $\mathcal{C}$ is left semisimple.

The connection to Galois corings is the following, due to Wisbauer [41, 3.12]:

Proposition 3.10 For an A-coring with a fixed grouplike element $(\mathcal{C}, x)$, the following assertions are equivalent:

1. $\mathcal{C}$ is a simple and left (or right) semisimple coring;

2. $(\mathcal{C}, x)$ is Galois and $\operatorname{End}^{\mathcal{C}}(A)$ is simple and left semisimple;

3. $(\mathcal{C}, x)$ is Galois and $B$ is a simple left semisimple subring of $A$;

4. $\mathcal{C}$ is flat as a right $A$-module, $A$ is a projective generator in ${ }^{\mathcal{C}} \mathcal{M}$, and $\operatorname{End}^{\mathcal{C}}(A)$ is simple and left semisimple (the left $\mathcal{C}$-coaction on $A$ being given by $\left.\rho^{l}(a)=a x\right)$.

\section{Galois corings and Morita theory}

Let $(\mathcal{C}, x)$ be a coring with a fixed grouplike element, $B=A^{\text {coC }}$, and $\mathcal{D}=$ $A \otimes_{B} A$. We can consider the left dual of the map can:

$$
{ }^{*} \text { can }:{ }^{*} \mathcal{C} \rightarrow{ }^{*} \mathcal{D} \cong{ }_{B} \operatorname{End}(A)^{\mathrm{op}},{ }^{*} \operatorname{can}(f)(a)=f(x a) .
$$

The following result is obvious.

Proposition 4.1 If $(\mathcal{C}, x)$ is Galois, then ${ }^{*}$ can is an isomorphism. The converse property holds if $\mathcal{C}$ and $A$ are finitely generated projective, respectively as a left $A$-module, and a left $B$-module.

Let $Q=\left\{q \in{ }^{*} \mathcal{C} \mid c_{(1)} q\left(c_{(2)}\right)=q(c) x\right.$, for all $\left.c \in \mathcal{C}\right\}$. A straightforward computation shows that $Q$ is a $\left({ }^{*} \mathcal{C}, B\right)$-bimodule. Also $A$ is a left $\left(B,{ }^{*} \mathcal{C}\right)$-bimodule; the right ${ }^{*} \mathcal{C}$-action is induced by the right $\mathcal{C}$-coaction: $a \cdot f=f(x a)$. Now consider the maps

$$
\begin{gathered}
\tau: A \otimes_{*_{\mathcal{C}}} Q \rightarrow B, \tau\left(a \otimes_{*_{\mathcal{C}}} q\right)=q(x a) ; \\
\mu: Q \otimes_{B} A \rightarrow{ }^{*} \mathcal{C}, \mu\left(q \otimes_{B} a\right)=q \# i(a) .
\end{gathered}
$$

With this notation, we have the following property (see 14]).

Proposition $4.2\left(B,{ }^{*} \mathcal{C}, A, Q, \tau, \mu\right)$ is a Morita context.

Properties of this Morita context are studied in [1, 2], 14] and [15. It generalizes (and unifies) Morita contexts discussed in [5], 17, [19], 20] and 23. We recall the following properties from [14] and [15].

Proposition 4.3 14, Th. 3.3 and Cor. 3.4] If $\tau$ is surjective, then $M^{\mathrm{coC}}=$ $M^{*} \mathcal{C}=\left\{m \in M \mid m \cdot f=m f(x)\right.$, for all $\left.f \in{ }^{*} \mathcal{C}\right\}$, for all $M \in \mathcal{M}^{\mathcal{C}}$.

The following assertions are equivalent:

1. $\tau$ is surjective;

2. there exists $q \in Q$ such that $q(x)=1$;

3. for every $M \in \mathcal{M}^{*} \mathcal{C}$, the map

$$
\omega_{M}: M \otimes *_{\mathcal{C}} Q \rightarrow M^{* \mathcal{C}}, \omega_{M}\left(m \otimes *_{\mathcal{C}} q\right)=m \cdot q
$$

is bijective.

Proposition 4.4 [15, 14, Th. 3.5] The following assertions are equivalent:

1. $\mu$ is surjective;

2. $\mathcal{C}$ is finitely generated and projective as a left $A$-module and $G$ is fully faithful.

As an application of Proposition 4.3 we have the following result. 
Corollary 4.5 Assume that $\mathcal{C}$ is finitely generated projective as a left $A$ module. Consider the adjoint pair $\left(F=\bullet \otimes_{B} A, G=(\bullet)^{\mathrm{co} \mathcal{C}}\right)$, and the functors $\widetilde{F}=\bullet \otimes_{B} A$ and $\widetilde{G}=\bullet \otimes_{*_{\mathcal{C}}} Q$ coming from the Morita context of Proposition 4.2 Then $F \cong \widetilde{F}$ and $G \cong \widetilde{G}$ if $\tau$ is surjective.

Proof Take $N \in \mathcal{M}_{B} . F(N)$ corresponds to $\widetilde{F}$ under the isomorphism $\mathcal{M}^{\mathcal{C}} \cong$ $\mathcal{M}_{*}$ C. If $\tau$ is surjective, then it follows from Proposition 4.3 that $\omega: \widetilde{G} \rightarrow G$ is bijective.

Let us now compute the Morita context associated to the canonical coring.

Proposition 4.6 Let $i: B \rightarrow A$ be a ring morphism, and assume that $i$ is pure as a morphism of left $B$-modules. Then the Morita context associated to the canonical coring $\left(\mathcal{D}=A \otimes_{B} A, 1 \otimes_{B} 1\right)$ is the Morita context $\left(B,{ }_{B} \operatorname{End}(A)^{\mathrm{op}}, A\right.$, $\left.{ }_{B} \operatorname{Hom}(A, B), \varphi, \psi\right)$ associated to $A$ as a left $B$-module (see [4, II.4]).

Proof From Proposition 2.1] it follows that

$$
A^{\mathrm{coD}}=\left\{b \in A \mid b \otimes_{B} 1=1 \otimes_{B} b\right\}=B .
$$

Take $q \in Q \subset{ }_{A} \operatorname{Hom}\left(A \otimes_{B} A, A\right)$ and the corresponding $\widetilde{q} \in{ }_{B} \operatorname{Hom}(A, A)$, given by $\tilde{q}(a)=q\left(1 \otimes_{B} a\right)$. Then

$$
q\left(a^{\prime} \otimes_{B} a\right)\left(1 \otimes_{B} 1\right)=\left(a^{\prime} \otimes_{B} 1\right) q\left(1 \otimes_{B} a\right) .
$$

Taking $a^{\prime}=1$, we find

$$
\tilde{q}(a) \otimes_{B} 1=1 \otimes_{B} \tilde{q}(a)
$$

hence $\tilde{q}(a) \in B$, and it follows that $Q \subset{ }_{B} \operatorname{Hom}(A, B)$. The converse inclusion is proved in a similar way. A straightforward verification shows that $\varphi=\tau$ and $\psi=\mu$.

Recall that the context associated to the left $A$-module $B$ is strict if and only if $A$ is a left $B$-progenerator. We are now ready to prove the following result. In Section [5 we will see that it is a generalization of [17. Th. 9.3 and 9.6].

Theorem 4.7 Let $(\mathcal{C}, x)$ be a coring with fixed grouplike element, and assume that $\mathcal{C}$ is a left $A$-progenerator. We take a subring $B^{\prime}$ of $B=A^{\mathrm{coC}}$, and consider the map

$$
\operatorname{can}^{\prime}: \mathcal{D}^{\prime}=A \otimes_{B^{\prime}} A \rightarrow \mathcal{C}, \operatorname{can}^{\prime}\left(a \otimes_{B^{\prime}} a^{\prime}\right)=a x a^{\prime}
$$

Then the following statements are equivalent:

1. - can' $^{\prime}$ is an isomorphism;

- A is faithfully flat as a left $B^{\prime}$-module.

2. $\quad{ }^{*} \mathrm{can}^{\prime}$ is an isomorphism;

- A is a left $B^{\prime}$-progenerator.

3. $\bullet B=B^{\prime}$;

- the Morita context $\left(B,{ }^{*} \mathcal{C}, A, Q, \tau, \mu\right)$ is strict.

4. $\quad B=B^{\prime}$;

- $(F, G)$ is an equivalence of categories.

Proof 1$) \Leftrightarrow 2$ ). Obviously ${ }^{*} \mathrm{can}^{\prime}$ is an isomorphism if $\mathrm{can}^{\prime}$ is an isomorphism, and the converse holds if $\mathcal{C}$ is a left $A$-progenerator and $A$ is a left $B^{\prime}$-progenerator. If $\mathrm{can}^{\prime}$ is an isomorphism, then $A \otimes_{B^{\prime}} A=\mathcal{D}^{\prime} \cong \mathcal{C}$ is a left $A$-progenerator, hence $A$ is a left $B^{\prime}$-progenerator.

$1) \Rightarrow 3$ ). Since $A$ is faithfully flat as a left $B^{\prime}$-module, $A^{\text {co } \mathcal{D}^{\prime}}=B^{\prime}$. Since can ${ }^{\prime}$ is an isomorphism, it follows that $B=A^{\mathrm{coC}}=A^{\mathrm{co} \mathcal{D}^{\prime}}=B^{\prime}$. Then can $=\mathrm{can}^{\prime}$ is 
an isomorphism, hence the Morita contexts associated to $(\mathcal{C}, x)$ and $\left(\mathcal{D}, 1 \otimes_{B} 1\right)$ are isomorphic. From the equivalence of 1) and 2), we know that $A$ is a left $B$ progenerator, so the context associated to $\left(\mathcal{D}, 1 \otimes_{B} 1\right)$ is strict, see the remark preceding Theorem 4.7 Therefore the Morita context $\left(B,{ }^{*} \mathcal{C}, A, Q, \tau, \mu\right)$ associated to $(\mathcal{C}, x)$ is also strict.

$3) \Rightarrow 1)$. and 3$) \Rightarrow 4)$. If $\left(B,{ }^{*} \mathcal{C}, A, Q, \tau, \mu\right)$ is strict, then $A$ is a left $B$-progenerator, and a fortiori faithfully flat as a left $B$-module. $\tau$ is surjective, so it follows from Corollary 4.5 that $F \cong \widetilde{F}$ and $G \cong \widetilde{G}$. $(\widetilde{F}, \widetilde{G})$ is an equivalence, so $(F, G)$ is also an equivalence. Then $(\mathcal{C}, x)$ is Galois by Proposition 3.1

$4) \Rightarrow 1$ ). can is an isomorphism, by Proposition 3.1 and we have already seen that this implies that $A$ is a left $B$-progenerator, so $A$ is faithfully flat as a left $B$-module.

\section{Application to particular cases}

5.1 Coalgebra Galois extensions. From [10, we recall the following Definition.

Definition 5.1 Let $i: B \rightarrow A$ be a morphism of $k$-algebras, and $C$ a $k$ coalgebra. $A$ is called a $C$-Galois extension of $B$ if the following conditions hold:

1. $A$ is a right $C$-comodule;

2. can : $A \otimes_{B} A \rightarrow A \otimes C, \operatorname{can}\left(a \otimes_{B} a^{\prime}\right)=a a_{[0]}^{\prime} \otimes_{B} a_{[1]}^{\prime}$ is an isomorphism;

3. $B=\{a \in A \mid \rho(a)=a \rho(1)\}$.

Proposition 5.2 Let $i: B \rightarrow A$ be a morphism of $k$-algebras, and $C$ a $k$-coalgebra. $A$ is called a $C$-Galois extension of $B$ if and only if there exists a right-right entwining structure $(A, C, \psi)$ and $x \in G(A \otimes C)$ such that $A^{\mathrm{co} A \otimes C}=B$ and $(A \otimes C, x)$ is a Galois coring.

Proof Let $(A, C, \psi)$ be an entwining structure. We have seen in Example 1.6 that $\mathcal{C}=A \otimes C$ is a coring. Given a grouplike element $x=\sum_{i} a_{i} \otimes c_{i}$, we have a right $\mathcal{C}$-coaction on $A$, hence $A$ is an entwined module (see Example 1.6), and therefore a $C$-comodule. The $C$-coaction is given by the formula

$$
\rho(a)=a_{[0]} \otimes a_{[1]}=\sum_{i} a_{i} a_{\psi} \otimes c_{i}^{\psi} .
$$

Then the conditions of Definition 5.1 are satisfied, and $A$ is $C$-Galois extension of $B$.

Conversely, let $A$ be a $C$-Galois extension of $B$. can is bijective, so the coring structure on $A \otimes_{B} A$ induces a coring structure on $A \otimes C$. We will show that this coring structure comes from an entwining structure $(A, C, \psi)$.

It is clear that the natural left $A$-module structure on $A \otimes C$ makes can into a left $A$-linear map. The right $A$-module structure on $A \otimes C$ induced by can is given by

$$
(b \otimes c) a=\operatorname{can}\left(\operatorname{can}^{-1}(b \otimes c) a\right) .
$$

Since $\operatorname{can}^{-1}\left(1_{[0]} \otimes 1_{[1]}\right)=1 \otimes_{B} 1$, we have

$$
\left(1_{[0]} \otimes 1_{[1]}\right) a=\operatorname{can}(1 \otimes a)=a_{[0]} \otimes a_{[1]} .
$$

The comultiplication $\Delta$ on $A \otimes C$ is given by

$$
\Delta(a \otimes c)=\left(\operatorname{can} \otimes_{A} \operatorname{can}\right) \Delta_{\mathcal{C}}\left(\operatorname{can}^{-1}(a \otimes c)\right) \in(A \otimes C) \otimes_{A}(A \otimes C),
$$


for all $a \in A$ and $c \in C$. can is bijective, so we can find $a_{i}, b_{i} \in A$ such that

$$
\operatorname{can}\left(\sum_{i} a_{i} \otimes_{B} b_{i}\right)=\sum_{i} a_{i} b_{i[0]} \otimes_{B} b_{i[1]}=a \otimes c,
$$

and we compute that

$$
\begin{aligned}
\Delta(a \otimes c) & =\left(\operatorname{can} \otimes_{A} \operatorname{can}\right) \Delta_{\mathcal{C}}\left(\sum_{i} a_{i} \otimes_{B} b_{i}\right) \\
& =\sum_{i} \operatorname{can}\left(a_{i} \otimes_{B} 1\right) \otimes_{A} \operatorname{can}\left(1 \otimes_{B} b_{i}\right) \\
& =\sum_{i}\left(a_{i} 1_{[0]} \otimes 1_{[1]}\right) \otimes_{A}\left(b_{i[0]} \otimes b_{i[1]}\right) \\
& =\sum_{i}\left(a_{i} 1_{[0]} \otimes 1_{[1]}\right) b_{i[0]} \otimes_{A}\left(1 \otimes b_{i[1]}\right) \\
\text { [5.1] } & =\sum_{i}\left(a_{i} b_{i[0]} \otimes b_{i[1]}\right) \otimes_{A}\left(1 \otimes b_{i[2]}\right) \\
& =\left(a \otimes c_{(1)}\right) \otimes_{A}\left(1 \otimes c_{(2)}\right) .
\end{aligned}
$$

Finally

$$
\begin{aligned}
\varepsilon_{C} c(a \otimes c) & =\varepsilon_{\mathcal{C}}\left(\sum_{i} a_{i} \otimes b_{i}\right)=\sum_{i} a_{i} b_{i} \\
& =\sum_{i} a_{i} b_{i[0]} \varepsilon_{C}\left(b_{i[1]}\right)=a \varepsilon_{C}(c) .
\end{aligned}
$$

Now define $\psi: C \otimes A \rightarrow A \otimes C$ by $\psi(c \otimes a)=\left(1_{A} \otimes c\right) a$. It follows from 9 that $(A, C, \psi)$ is an entwining structure.

Let $(A, C, \psi)$ be an entwining structure, and consider $g \in C$ grouplike. Then $x=1_{A} \otimes g$ is a grouplike element of $A \otimes C$. Let us first describe the Morita context from the previous Section.

Observe that ${ }^{*} \mathcal{C}={ }_{A} \operatorname{Hom}(A \otimes C, A) \cong \operatorname{Hom}(C, A)$ as a $k$-module. The ring structure on ${ }^{*} \mathcal{C}$ induces a $k$-algebra structure on $\operatorname{Hom}(C, A)$, and this $k$-algebra is denoted $\#(C, A)$. The product is given by the formula

$$
(f \# g)(c)=f\left(c_{(2)}\right)_{\psi} g\left(c_{(1)}^{\psi}\right) .
$$

We have a natural algebra homomorphism $i: A \rightarrow \#(C, A), i(a)(c)=\varepsilon_{C}(c) a$, and we have, for all $a \in A$ and $f: C \rightarrow A$ :

$$
(i(a) \# f)(c)=a_{\psi} f\left(c^{\psi}\right) \text { and }(f \# i(a))(c)=f(c) a .
$$

$\operatorname{Hom}(C, A)$ will denote the $k$-algebra with the usual convolution product, that is

$$
(f * g)(c)=f\left(c_{(1)}\right) g\left(c_{(2)}\right) .
$$

The ring of coinvariants is

$$
B=A^{\operatorname{co} C}=\left\{b \in A \mid b_{\psi} \otimes g^{\psi}=b \otimes g\right\},
$$

and the bimodule $Q$ is naturally isomorphic to

$$
Q=\left\{q \in \#(C, A) \mid q\left(c_{(2)}\right)_{\psi} \otimes c_{(1)}^{\psi}=q(c) \otimes g\right\} .
$$

We have maps

$$
\begin{gathered}
\mu: Q \otimes_{B^{\prime}} A \rightarrow \#(C, A), \mu\left(q \otimes_{B} a\right)(c)=q(c) a, \\
\tau: A \otimes_{\#(C, A)} Q \rightarrow B, \tau(a \otimes q)=a_{\psi} q\left(x^{\psi}\right),
\end{gathered}
$$


and $(B, \#(C, A), A, Q, \tau, \mu)$ is a Morita context.

Proposition 5.3 14 Prop. 4.3] Assume that $\lambda: C \rightarrow A$ is convolution invertible, with convolution inverse $\lambda^{-1}$. Then the following assertions are equivalent:

1. $\lambda \in Q$;

2. for all $c \in C$, we have

$$
\lambda^{-1}\left(c_{(1)}\right) \lambda\left(c_{(3)}\right)_{\psi} \otimes c_{(2)}^{\psi}=\varepsilon(c) 1_{A} \otimes g ;
$$

3. for all $c \in C$, we have

$$
\lambda^{-1}\left(c_{(1)}\right) \otimes c_{(2)}=\lambda^{-1}(c)_{\psi} \otimes g^{\psi} .
$$

Notice that condition 3) means that $\lambda^{-1}$ is right $C$-colinear. If such a $\lambda \in Q$ exists, then we call $(A, C, \psi, g)$ cleft.

Proposition 5.4 14 Prop. 4.4] Assume that $(A, C, \psi, g)$ is a cleft entwining structure. Then the map $\tau$ in the associated Morita context is surjective.

We say that the entwining structure $(A, C, \psi, g)$ satisfies the right normal basis property if there exists a left $B$-linear and right $C$-colinear isomorphism $B \otimes C \rightarrow A$. The following is one of the main results in [14. As before, we consider the functor $F=\bullet \otimes_{B} A: \mathcal{M}_{B} \rightarrow \mathcal{M}(\psi)_{A}^{C}$ and its right adjoint $G=(\bullet)^{\operatorname{co} C}$.

Theorem 5.5 14 Theorem 4.5] Let $(A, C, \psi, g)$ be an entwining structure with a fixed grouplike element. The following assertions are equivalent:

1. $(A, C, \psi, g)$ is cleft;

2. $(F, G)$ is a category equivalence and $(A, C, \psi, g)$ satisfies the right normal basis property;

3. $(A, C, \psi, g)$ is Galois, and satisfies the right normal basis property;

4. the map ${ }^{*}$ can : $\#(C, A) \rightarrow \operatorname{End}_{B}(A)^{\mathrm{op}}$ is bijective and $(A, C, \psi, g)$ satisfies the right normal basis property.

5.2 Hopf-Galois extensions. Let $H$ be a Hopf algebra over a commutative ring $k$ with bijective antipode, and $A$ a right $H$-comodule algebra (cf. Example 1.5). Then $\mathcal{C}=A \otimes H$ is an $A$-coring, and $1_{A} \otimes 1_{H} \in G(\mathcal{C})$. Let $B=A^{\text {co } H}$. The canonical map is now the following:

$$
\text { can : } A \otimes_{B} A \rightarrow A \otimes H, \operatorname{can}\left(a^{\prime} \otimes_{B} a\right)=a^{\prime} a_{[0]} \otimes a_{[1]}
$$

Definition 5.6 (see e.g. 24. Def. 1.1]) $A$ is a Hopf-Galois extension of $B$ if and only if can is an isomorphism.

Obviously $A$ is a Hopf-Galois extension of $B$ if and only if $\left(A \otimes H, 1_{A} \otimes 1_{H}\right)$ is a Galois coring.

Assume now that $H$ is a progenerator as a $k$-module, i.e. $H$ is finitely generated, faithful, and projective as a $k$-module. Then $A \otimes H$ is a left $A$-progenerator, so we can apply the results of Section 4 We will show that we recover results from 17. To this end, we will describe the Morita context associated to $\left(A \otimes H, 1_{A} \otimes 1_{H}\right)$. First we compute ${ }^{*} \mathcal{C}$. We have already seen in Section 5.1 that ${ }^{*} \mathcal{C} \cong \#(H, A)$. As a module, $\operatorname{Hom}(H, A) \cong H^{*} \otimes A$, since $H$ is finitely generated and projective. The multiplication on $\#(H, A)$ can be transported into a multiplication on $H^{*} \otimes A$, giving us a $k$-algebra denoted by $H^{*} \# A$. A straightforward computation shows that this multiplication is given by the following formula. $H^{*}$ is a coalgebra, since $H$ is 
finitely generated projective, and $H^{*}$ acts on $A$ from the left: $h^{*} \rightarrow a=\left\langle h^{*}, a_{[1]}\right\rangle a_{[0]}$. Then we can compute that

$$
\left(h^{*} \# a\right)\left(k^{*} \# b\right)=\left(k_{(1)}^{*} * h^{*}\right) \#\left(k_{(2)}^{*} \rightarrow a_{[0]}\right) b .
$$

Consider the map can' $: A \otimes A \rightarrow A \otimes H$; its dual ${ }^{*} \operatorname{can}^{\prime}: H^{*} \# A \rightarrow \operatorname{End}(A)^{\mathrm{op}}$ is given by

$$
{ }^{*} \operatorname{can}^{\prime}\left(h^{*} \# a\right)(b)=\left(h^{*}-b\right) a .
$$

Take $y=\sum_{i} h_{i}^{*} \# a_{i} \in H^{*} \# A . y \in Q$ if and only if

$$
\sum_{i}\left\langle h_{i}^{*}, h_{(2)}\right\rangle a_{i[0]} \otimes h_{(1)} a_{i[1]}=\sum_{i}\left\langle h_{i}^{*}, h\right\rangle a_{i} \otimes 1,
$$

for $h \in H$. Since $H$ is finitely generated and projective, this is also equivalent to

$$
\sum_{i}\left\langle h_{i}^{*}, h_{(2)}\right\rangle a_{i[0]}\left\langle h^{*}, h_{(1)} a_{i[1]}\right\rangle=\sum_{i}\left\langle h_{i}^{*}, h\right\rangle a_{i}\left\langle h^{*}, 1\right\rangle,
$$

for all $h \in H$ and $h^{*} \in H^{*}$. The left hand side equals

$$
\sum_{i}\left\langle h_{(1)}^{*} * h_{i}^{*}, h\right\rangle\left\langle h_{(2)}^{*}, a_{i[1]}\right\rangle a_{i[0]},
$$

so we find that $y \in Q$ if and only if

$$
\sum_{i}\left(h_{(1)}^{*} * h_{i}^{*}\right) \#\left(h_{(2)}^{*} \rightarrow a_{i}\right)=\left\langle h^{*}, 1\right\rangle \sum_{i} h_{i}^{*} \# a_{i},
$$

or

$$
y\left(h^{*} \# 1\right)=\left\langle h^{*}, 1\right\rangle y .
$$

for all $h^{*} \in H^{*}$. Thus

$$
Q=\left\{y \in H^{*} \# A \mid y\left(h^{*} \# 1\right)=\left\langle h^{*}, 1\right\rangle y, \text { for all } h^{*} \in H^{*}\right\} .
$$

Elementary computations show that the maps $\mu$ and $\tau$ from the Morita context are the following:

$$
\begin{gathered}
\tau: A \otimes_{H^{*} \# A} Q \rightarrow B, \tau(a \otimes y)={ }^{*} \operatorname{can}(y)(a) ; \\
\mu: Q \otimes_{B} A \rightarrow H^{*} \# A, \mu(y \otimes a)=y\left(\varepsilon_{C} \# a\right),
\end{gathered}
$$

where $B=A^{\mathrm{co} H}$, as usual. Theorem 4.7 now takes the following form.

Proposition 5.7 Let $H$ be a k-progenerator Hopf algebra over a commutative ring $k$, and $A$ a right $H$-comodule algebra. Then the following statements are equivalent (with notation as above):

1. - $\operatorname{can}^{\prime}: A \otimes A \rightarrow A \otimes H, \operatorname{can}^{\prime}\left(a^{\prime} \otimes a\right)=a^{\prime} a_{[0]} \otimes a_{[1]}$ is bijective;

- $A$ is faithfully flat as a $k$-module.

2. $\quad{ }^{*} \operatorname{can}^{\prime}: H^{*} \# A \rightarrow \operatorname{End}(A)^{\mathrm{op}},{ }^{*} \operatorname{can}\left(h^{*} \# a\right)(b)=\left(h^{*}-b\right) a$ is an isomorphism;

- $A$ is a k-progenerator.

3. $\quad A^{\mathrm{co} H}=k$;

- the Morita context $\left(k, H^{*} \# A, A, Q, \tau, \mu\right)$ is strict.

4. $\quad A^{\mathrm{co} H}=k$;

- the adjoint pair of functors $\left(F=\bullet \otimes A, G=(\bullet)^{\mathrm{co} H}\right)$ is an equivalence between the categories $\mathcal{M}_{k}$ and $\mathcal{M}_{A}^{H}$.

17. Theorems 9.3 and 9.6] follow from Proposition 5.7 
5.3 Classical Galois Theory. As in Example 1.3 let $G$ be a finite group, and $A$ a $G$-module algebra. We have seen that $\mathcal{C}=A \otimes(k G)^{*}=\oplus_{\sigma \in G} A v_{\sigma}$ is an $A$-coring. $\sum_{\sigma} v_{\sigma}$ is a grouplike element. Since $(k G)^{*}$ is finitely generated and projective, we can apply Proposition 5.7 We have

$$
\begin{gathered}
\operatorname{can}^{\prime}: A \otimes A \rightarrow \oplus_{\sigma \in G} A v_{\sigma}, \operatorname{can}^{\prime}(a \otimes b)=\sum_{\sigma} a \sigma(b) v_{\sigma} . \\
{ }^{*} \mathcal{C}=\oplus_{\sigma} u_{\sigma} A,
\end{gathered}
$$

with multiplication

$$
\left(u_{\sigma} a\right)\left(u_{\tau} b\right)=u_{\tau \sigma} \tau(a) b
$$

and

$$
{ }^{*} \operatorname{can}^{\prime}: \oplus_{\sigma} u_{\sigma} A \rightarrow \operatorname{End}(A)^{\mathrm{op}},{ }^{*} \operatorname{can}^{\prime}\left(u_{\sigma} a\right)(b)=\sigma(b) a .
$$

We also have

$$
Q=\left\{\sum_{\sigma} u_{\sigma} \sigma(a) \mid a \in A\right\} \cong A,
$$

which is not surprising since $(k G)^{*}$ is a Frobenius Hopf algebra (see [14] and 20]). If $A^{G}=k$, then we have a Morita context $\left(k,{ }^{*} \mathcal{C}, A, A, \tau, \mu\right)$, where the connecting maps are the following:

$$
\begin{gathered}
\tau: A \otimes *_{\mathcal{C}} A \rightarrow k, \tau(a \otimes b)=\sum_{\sigma} \sigma(a b) \\
\mu: A \otimes A \rightarrow{ }^{*} \mathcal{C}, \mu(a \otimes b)=\sum_{\sigma} u_{\sigma} \sigma(b) a .
\end{gathered}
$$

Proposition 5.7 now takes the following form (compare to [21 Prop. III.1.2]).

Proposition 5.8 Let $G$ be a finite group, $k$ a commutative ring and $A$ a $G$ module algebra. Then the following statements are equivalent:

1. $\quad$ can' is an isomorphism;

- A is faithfully flat as a $k$-module.

2. $\quad$ - ${ }^{*} \operatorname{can}^{\prime}$ is an isomorphism;

- A is a k-progenerator.

3. $\quad A^{G}=k$;

- the Morita context $\left(k,{ }^{*} \mathcal{C}, A, A, \tau, \mu\right)$ is strict.

4. $\quad A^{G}=k$;

- the adjoint pair of functors $\left(F=\bullet \otimes A, G=(\bullet)^{G}\right)$ is an equivalence between the categories of $k$-modules and right $A$-modules on which $G$ acts as a group of right A-semilinear automorphisms.

In the case where $A$ is a commutative $G$-module algebra, we have some more equivalent conditions.

Proposition 5.9 Let $G$ be a finite group, $k$ a commutative ring and $A$ a commutative $G$-module algebra. Then the statements of Proposition 5.8 are equivalent to

5. $\quad A^{G}=k$;

- for each non-zero idempotent $e \in S$ and $\sigma \neq \tau \in G$, there exists $a \in A$ such that $\sigma(a) e \neq \tau(a) e$;

6. $\bullet A^{G}=k$; 
- there exist $x_{1}, \cdots, x_{n}, y_{1}, \cdots y_{n} \in A$ with

$$
\sum_{j=1}^{n} x_{j} \sigma\left(y_{j}\right)=\delta_{\sigma, e}
$$

for all $\sigma \in G$.

7. $\bullet A^{G}=k$;

- for each maximal ideal $m$ of $A$, and for each $\sigma \neq e \in G$, there exists $x \in S$ such that $\sigma(x)-x \notin m$.

Proof We refer to [16. Th 1.3] and [21, Prop. III.1.2].

If $A=l$ is a field, then the second part of condition 7. is satisfied. Let $l$ be a finite field extension of a field $k$, and $G$ the group of $k$-automorphisms of $l$. Then $l^{G}=k$ if and only if $l$ is a normal and separable (in the classical sense) extension of $k$ (see e.g. [37, Th. 10.8 and 10.10]). Thus we recover the classical definition of a Galois field extension.

5.4 Strongly graded rings. As in Example 1.4 let $G$ be a group, and $A$ a $G$-graded ring, and $\mathcal{C}=\oplus_{\sigma \in G} A u_{\sigma}$. Fix $\lambda \in G$, and take the grouplike element $u_{\lambda} \in G(\mathcal{C})$. Then $M^{\mathrm{coC}}=M_{\lambda}$, for any right $G$-graded $A$-module, and $B=A^{\mathrm{coC}}=$ $A_{e}$. Since $B$ is a direct factor of $A, A$ is flat as a left and right $B$-module, and $i: B \rightarrow A$ is pure in $\mathcal{M}_{B}$ and ${ }_{B} \mathcal{M}$. Also

$$
\text { can : } A \otimes_{B} A \rightarrow \oplus_{\sigma \in G} A u_{\sigma}, \operatorname{can}\left(a^{\prime} \otimes a\right)=\sum_{\sigma \in G} a^{\prime} a_{\sigma} u_{\lambda \sigma} .
$$

Proposition 5.10 With notation as above, the following assertions are equivalent.

1. A is strongly $G$-graded, that is, $A_{\sigma} A_{\tau}=A_{\sigma \tau}$, for all $\sigma, \tau \in G$;

2. the pair of adjoint functors $\left(F=\bullet \otimes_{B} A, G=(\bullet)_{\lambda}\right)$ is an equivalence between $\mathcal{M}_{B}$ and $\mathcal{M}_{A}^{G}$, the category of $G$-graded right $A$-modules;

3. $\left(\mathcal{C}, u_{\lambda}\right)$ is a Galois coring.

In this case $A$ is faithfully flat as a left (or right) $B$-module.

Proof 1$) \Rightarrow 2$ ) is a well-known fact from graded ring theory. We sketch a proof for completeness sake. The unit of the adjunction between $\mathcal{M}_{B}$ and $\mathcal{M}_{A}^{G}$ is given by

$$
\eta_{N}: N \rightarrow\left(N \otimes_{B} A\right)_{\lambda}, \eta_{N}(n)=n \otimes_{B} 1_{A} .
$$

$\eta_{N}$ is always bijective, even if $A$ is not strongly graded. Let us show that the counit maps $\zeta_{M}: M_{\lambda} \otimes_{B} A \rightarrow M, \zeta_{M}\left(m \otimes_{B} a\right)=m a$ are surjective. For each $\sigma \in G$, we can find $a_{i} \in A_{\sigma^{-1}}$ and $a_{i}^{\prime} \in A_{\sigma}$ such that $\sum_{i} a_{i} a_{i}^{\prime}=1$. Take $m \in M_{\tau}$ and put $\sigma=\lambda_{-1} \tau$. Then $m=\zeta_{M}\left(\sum_{i} m a_{i} \otimes_{B} a_{i}^{\prime}\right)$, and $\zeta_{M}$ is surjective.

If $m_{j} \in M_{\lambda}$ and $c_{j} \in A$ are such that $\sum_{j} m_{j} c_{j}=0$, then for each $\sigma \in G$, we have

$$
\sum_{j} m_{j} \otimes c_{j \sigma}=\sum_{i, j} m_{j} \otimes c_{j \sigma} a_{i} a_{i}^{\prime}=\sum_{i, j} m_{j} c_{j \sigma} a_{i} \otimes a_{i}^{\prime}=0 .
$$

hence $\sum_{j} m_{j} \otimes c_{j}=\sum_{\sigma \in G}=\sum_{j} m_{j} \otimes c_{j \sigma}=0$, so $\zeta_{M}$ is also injective.

2) $\Rightarrow 3$ ) follows from Proposition 3.1

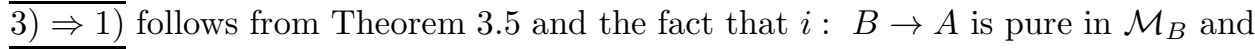
${ }_{B} \mathcal{M}$.

The final statement follows from Proposition 2.5] and the fact that $A$ is flat as a $B$-module. 
Notice that, in this situation, the fact that $\left(\mathcal{C}, u_{\lambda}\right)$ is Galois is independent of the choice of $\lambda$.

\section{A more general approach: comatrix corings}

Let $\mathcal{C}$ be an $A$-coring. We needed a grouplike $x \in \mathcal{C}$ in order to make $A$ into a right $\mathcal{C}$-comodule. In [25, the following idea is investigated. A couple $(\mathcal{C}, \Sigma)$, consisting of a coring $\mathcal{C}$ and a right $\mathcal{C}$-comodule $\Sigma$ which is finitely generated and projective as a right $A$-module, will be called a coring with a fixed finite comodule. Let $T=\operatorname{End}^{\mathcal{C}}(\Sigma)$. Then we have a pair of adjoint functors

$$
F=\bullet \otimes_{T} \Sigma: \mathcal{M}_{T} \rightarrow \mathcal{M}^{\mathcal{C}} ; G=\operatorname{Hom}^{\mathcal{C}}(\Sigma, \bullet): \mathcal{M}^{\mathcal{C}} \rightarrow \mathcal{M}_{T},
$$

with unit $\nu$ and counit $\zeta$ given by

$$
\begin{gathered}
\nu_{N}: N \rightarrow \operatorname{Hom}^{\mathcal{C}}\left(\Sigma, N \otimes_{T} \Sigma\right), \nu_{N}(n)(u)=n \otimes_{T} u \\
\zeta_{M}: \operatorname{Hom}^{\mathcal{C}}(\Sigma, M) \otimes_{T} \Sigma \rightarrow M, \zeta_{M}\left(f \otimes_{T} u\right)=f(u) .
\end{gathered}
$$

In the situation where $\Sigma=A$, we recover the adjoint pair discussed at the end of Section 11 A particular example is the comatrix coring, generalizing the canonical coring. Let $A$ and $B$ be rings, and $\Sigma \in{ }_{B} \mathcal{M}_{A}$, with $\Sigma$ finitely generated and projective as a right $A$-module. Let

$$
\left\{\left(e_{i}, e_{i}^{*}\right) \mid i=1, \cdots, n\right\} \subset \Sigma \times \Sigma^{*}
$$

be a finite dual basis of $\Sigma$ as a right $A$-module. $\mathcal{D}=\Sigma^{*} \otimes_{B} \Sigma$ is an $(A, A)$-bimodule, and an $A$-coring, via

$$
\Delta_{\mathcal{D}}\left(\varphi \otimes_{B} u\right)=\sum_{i} \varphi \otimes_{B} e_{i} \otimes_{A} e_{i}^{*} \otimes_{B} u \text { and } \varepsilon_{\mathcal{D}}\left(\varphi \otimes_{B} u\right)=\varphi(u) .
$$

Furthermore $\Sigma \in \mathcal{M}^{\mathcal{D}}$ and $\Sigma^{*} \in{ }^{\mathcal{D}} \mathcal{M}$. The coactions are given by

$$
\rho^{r}(u)=\sum_{i} e_{i} \otimes_{A} e_{i}^{*} \otimes_{B} u ; \rho^{l}(\varphi)=\sum_{i} \varphi \otimes_{B} e_{i} \otimes_{A} e_{i}^{*} .
$$

We also have that ${ }^{*} \mathcal{D} \cong{ }_{B} \operatorname{End}(\Sigma)^{\text {op }}$. El Kaoutit and Gómez Torrecillas proved the following generalization of the Faithfully Flat Descent Theorem.

Theorem 6.1 Let $\Sigma \in{ }_{B} \mathcal{M}_{A}$ be finitely generated and projective as a right $A$-module, and $\mathcal{D}=\Sigma^{*} \otimes \Sigma$. Then the following are equivalent

1. $\Sigma$ is faithfully flat as a left $B$-module;

2. $\Sigma$ is flat as a left $B$-module and $\left(\bullet \otimes_{B} \Sigma, \operatorname{Hom}^{\mathcal{D}}(\Sigma, \bullet)\right)$ is a category equivalence between $\mathcal{M}_{B}$ and $\mathcal{M}^{\mathcal{D}}$.

Let $(\mathcal{C}, \Sigma)$ be a coring with a fixed finite comodule, and $T=\operatorname{End}^{\mathcal{C}}(\Sigma)$. We have an isomorphism $f: \Sigma^{*} \rightarrow \operatorname{Hom}^{\mathcal{C}}(\Sigma, \mathcal{C})$ given by

$$
f(\varphi)=\left(\varphi \otimes_{A} I_{\mathcal{C}}\right) \circ \rho \text { and } f^{-1}(\phi)=\varepsilon_{\mathcal{C}} \circ \phi,
$$

for all $\varphi \in \Sigma^{*}$ and $\phi \in \operatorname{Hom}^{\mathcal{C}}(\Sigma, \mathcal{C})$. Consider the map

$$
\text { can }=\zeta_{\mathcal{C}} \circ\left(f \otimes_{B} I_{\Sigma}\right): \mathcal{D}=\Sigma^{*} \otimes_{T} \Sigma \rightarrow \operatorname{Hom}^{\mathcal{C}}(\Sigma, \mathcal{C}) \otimes_{T} \Sigma \rightarrow \mathcal{C} .
$$

We compute easily that $\operatorname{can}\left(\varphi \otimes_{B} u\right)=\varphi\left(u_{[0]}\right) u_{[1]}$. can is a morphism of corings, and can is an isomorphism if and only if $\zeta_{\mathcal{C}}$ is an isomorphism.

Definition 6.2 25, 3.4] Let $(\mathcal{C}, \Sigma)$ be a coring with a fixed finite comodule, and $T=\operatorname{End}^{\mathcal{C}}(\Sigma) .(\mathcal{C}, \Sigma)$ is termed Galois if can : $\Sigma^{*} \otimes_{T} \Sigma \rightarrow \mathcal{C}$ is an isomorphism. 
Theorem 6.3 25, 3.5] If $(\mathcal{C}, \Sigma)$ is Galois, and $A$ is faithfully flat as a left $T$-module, then $(F, G)$ is an equivalence of categories.

For further results, we refer to [25].

\section{ACKNOWLEDGEMENTS}

The author thanks George Janelidze for stimulating discussions about descent theory, and the referee for pointing out that the proper assumption in Proposition 2.3 is right purity of $i$, instead of left purity.

\section{References}

Abuhlail J. Morita contexts for corings and equivalences, in "Hopf algebras in non-commutative geometry and physics", Caenepeel S. and Van Oystaeyen, F. (eds.), Lecture Notes Pure Appl. Math., Dekker, New York, to appear.

Abuhlail, J. Rational modules for corings, preprint.

Auslander, M. and Goldman, O. The Brauer group of a commutative ring. Trans. Amer. Math. Soc. 97 (1960), 367-409.

Bass, H. "Algebraic K-theory", Benjamin, New York, 1968.

Beattie, M., Dăscălescu, S. and Raianu, Ş. Galois extensions for co-Frobenius Hopf algebras, J. Algebra 198 (1997), 164-183.

Böhm, G. Doi-Hopf modules over weak Hopf algebras, Comm. Algebra 28 (2000), 4687-4698.

Borceux, F. Handbook of categorical algebra 2, Encyclopedia Math. Appl. 51, Cambridge University Press, Cambridge, 1994.

Brzeziński, T. Coalgebra-Galois extensions from the extension point of view, in "Hopf algebras and quantum groups", Caenepeel S. and Van Oystaeyen, F. (eds.), Lecture Notes in Pure and Appl. Math. 209, Marcel Dekker, New York, 2000.

T. Brzeziński, The structure of corings. Induction functors, Maschke-type theorem, and Frobenius and Galois properties, Algebr. Representat. Theory 5 (2002), 389-410.

Brzeziński, T. and Hajac, P.M. Coalgebra extensions and algebra coextensions of Galois type, Comm. Algebra 27 (1999), 1347-1367.

Brzeziński, T. and Majid, S. Coalgebra bundles, Comm. Math. Phys. 191 (1998), 467-492.

Caenepeel, S. and De Groot, E. Modules over weak entwining structures, Contemp. Math. 267 (2000), 31-54.

Caenepeel, S., Militaru, G. and Zhu, Shenglin "Frobenius and separable functors for generalized module categories and nonlinear equations", Lecture Notes in Math. 1787, Springer Verlag, Berlin, 2002.

Caenepeel, S., Vercruysse, J. and Wang, Shuanhong Morita Theory for corings and cleft entwining structures, J. Algebra, to appear.

Caenepeel, S., Vercruysse, J. and Wang, Shuanhong Rationality properties for Morita contexts associated to corings, preprint.

Chase, S., Harrison, D. and Rosenberg, A. Galois theory and Galois cohomology of commutative rings, Mem. Amer. Math. Soc. 52 (1965), 1-19.

Chase, S. and Sweedler, M. E. "Hopf algebras and Galois theory", Lect. Notes in Math. 97, Springer Verlag, Berlin, 1969.

Cipolla, M. Discesa fedelmente piatta dei moduli, Rendiconti del Circolo Matematico di Palermo, Serie II 25 (1976).

Cohen, M. and Fischman, D. Semisimple extensions and elements of trace 1, J. Algebra 149 (1992), 419-437.

Cohen, M., Fischman, D. and Montgomery, S. Hopf Galois extensions, smash products, and Morita equivalence, J. Algebra 133 (1990), 351-372.

DeMeyer F. and Ingraham, E. "Separable algebras over commutative rings", Lecture Notes in Math. 181, Springer Verlag, Berlin, 1971.

Doi, Y. Unifying Hopf modules, J. Algebra 153 (1992), 373-385.

Doi, Y. Generalized smash products and Morita contexts for arbitrary Hopf algebras, in "Advances in Hopf algebras", Bergen, J. and Montgomery, S. (eds.), Lect. Notes Pure Appl. Math. 158, Dekker, New York, 1994.

Doi, Y. and Takeuchi, M. Hopf-Galois extensions of algebras, the Miyashita-Ulbrich action and Azumaya algebras, J. Algebra 121 (1989), 488-516. 
El Kaoutit, L. and Gómez Torrecillas, J. Comatrix corings: Galois corings, descent theory, and a structure Theorem for cosemisimple corings, Math. Z., to appear.

El Kaoutit, L., Gómez Torrecillas, J. and Lobillo, F. J. Semisimple corings, preprint 2001.

Gómez Torrecillas, J. Separable functors in corings, Int. J. Math. Math. Sci. 30 (2002), 203-225.

Grothendieck, A. Technique de Descente I, Sém. Bourbaki, exp. 190 (1959-1960).

Knus, M. A. and Ojanguren, M. "Théorie de la descente et algébres d'Azumaya", Lecture Notes in Math. 389, Springer Verlag, Berlin, 1974.

Koppinen, M. Variations on the smash product with applications to group-graded rings, J. Pure Appl. Algebra 104 (1995), 61-80.

Mac Lane, S. "Categories for the working mathematician", second edition, Graduate Texts in Mathematics 5, Springer Verlag, Berlin, 1997.

Mesablishvili, B. Pure morphisms of commutative rings are effective descent morphisms for modules - a new proof, Theory Appl. Categories 7 (2000), 38-42.

Nuss, P. Noncommutative descent and nonabelian cohomology, K-Theory, 12 (1997), 23-74.

Schauenburg, P. Doi-Koppinen modules versus entwined modules, New York J. Math., 6 (2000), 325-329.

Schneider, H.J. Principal homogeneous spaces for arbitrary Hopf algebras, Israel J. Math. 70 (1990), 167-195.

Serre, J.P. "Cohomologie Galoisienne", Lect. Notes in Math. 5, Springer Verlag, Berlin, 1965.

Stewart, I. "Galois theory", second edition, Chapman and Hall, London, 1989.

Sweedler, M. E. The predual Theorem to the Jacobson-Bourbaki Theorem, Trans. Amer. Math. Soc. 213 (1975), 391-406.

Takeuchi, M., as referred to in MR 2000c 16047, by A. Masuoka.

Wisbauer, R. On the category of comodules over corings, in "Mathematics and mathematics education (Bethlehem, 2000)", World Sci. Publishing, River Edge, NJ, 2002, 325-336.

Wisbauer, R. On Galois corings, in "Hopf algebras in non-commutative geometry and physics", Caenepeel S. and Van Oystaeyen, F. (eds.), Lecture Notes Pure Appl. Math., Dekker, New York, to appear. 